\title{
Nuclear Spin Relaxation for Higher Spin
}

\author{
W. Apel ${ }^{1}$ and Yu. A. Bychkov ${ }^{2,1}$ \\ 1 Physikalisch-Technische Bundesanstalt, Bundesallee 100, 38116 Braunschweig, Germany. \\ ${ }^{2}$ L.D. Landau Institute for Theoretical Physics, ul.Kosigina, 2, Moscow, Russia.
}

(November 23, 2018)

\begin{abstract}
We study the relaxation of a spin $I$ that is weakly coupled to a quantum mechanical environment. Starting from the microscopic description, we derive a system of coupled relaxation equations within the adiabatic approximation. These are valid for arbitrary $I$ and also for a general stationary non-equilibrium state of the environment. In the case of equilibrium, the stationary solution of the equations becomes the correct Boltzmannian equilibrium distribution for given spin $I$. The relaxation towards the stationary solution is characterized by a set of relaxation times, the longest of which can be shorter, by a factor of up to $2 I$, than the relaxation time in the corresponding Bloch equations calculated in the standard perturbative way.
\end{abstract}

Nuclear magnetic resonance is a well-established method for testing electronic properties in solids [1]. In recent years, it became possible to apply this technique not only in three dimensions, but also to a two-dimensional electron system, the quantum Hall ferromagnet that is realized in semiconductor heterostructures in a strong magnetic field. The experimental work lead to the unexpected conclusion that a new kind of low-energy states, Skyrmions, can be formed and can determine the nuclear relaxation processes in these systems when one Landau sub-level of one spin direction is filled [2 11].

As a theoretical description of spin relaxation, Bloch's equations have been successfully used for about fifty years now. While these phenomenological equations are applicable in a wide range of cases, their microscopic derivation reveals two main restrictions. First, as was already discussed in the original work [12], the derivation becomes strictly valid, if either the spin is $I=1 / 2$ or the temperature of the bath is large compared to the resonance frequency. But the spin in the system under study can be $I=3 / 2$ (for ${ }^{69} \mathrm{Ga},{ }^{71} \mathrm{Ga}$, and ${ }^{75} \mathrm{As}$; cf. Ref. [2]), or higher in the case of magnetic impurities. Further, the progress in the experimental techniques now lets a regime of temperatures and magnetic fields come into reach, in which the temperature of the bath is of the same order as the nuclear resonance energy (nuclear Zeeman energy). The second restriction in the derivation of the phenomenological equations demands that the environment (bath), the quantum mechanical degrees of freedom causing the spin relaxation, be in thermodynamic equilibrium. But in the case of the quantum Hall ferromagnet, the nuclear spins are coupled to a two-dimensional electron gas in which the electron-electron interaction plays the key role, since all single particle states are degenerate into a single Landau level due to the strong magnetic field; such a system, dominated by Skyrmionstates, is not necessarily in equilibrium. Thus, it appears worthwhile to reconsider the microscopic derivation of phenomenological equations for the spin relaxation in order to investigate whether there is a significant difference between the general case and a case in which the Bloch equations are valid.
It is the purpose of this work to relax the two above restrictions by investigating the general case of an arbitrary spin $I$ and also an arbitrary stationary state of the environment responsible for the relaxation. We refrain from studying a specific mechanism and consider instead the general case of a magnetic moment coupled to a bath of other quantum degrees of freedom. This magnetic moment can be a nuclear spin, or also a magnetic impurity. In the following, we use the terms "nuclear spin" for the magnetic moment and "electrons" for the bath - usually the latter is called "lattice". Then, the contribution of the nuclear spin to the Hamiltonian is

$$
H=-\gamma \hat{\vec{I}} \cdot\left(\vec{B}_{0}+\hat{\vec{B}}\right) .
$$

Here, the magnetic moment, $\gamma \hat{\vec{I}}$ (where $\hat{\vec{I}}$ is the spin), couples to an effective magnetic field. It is well known that for $I>1 / 2$, there is an additional term causing relaxation, the electric quadrupole moment of the nucleus coupled to an inhomogeneous external electric field. Here, we have omitted this term in $H$, since the model Eq. (11) already suffices for the demonstration of our method; an inclusion of a quadrupolar coupling is straightforward. The effective magnetic field acting on the nuclear spin $\hat{\vec{I}}$ in Eq. (1) contains an operator $\hat{\vec{B}}$ of the electronic system. One can picture $\hat{\vec{B}}$ as being proportional to the electrons' spin. Its longitudinal component, $\hat{B}_{z}$, modifies the eigenvalues of the nuclear spin system, while the transverse components cause transitions between eigenstates. There is also a fixed part of the magnetic field in $z$-direction, $\vec{B}_{0}=B_{0} \hat{e}_{z}$, which acts as an external field. The coupling beween nuclear spin and electrons is supposed to be weak in the sense that we can use the adiabatic approximation as discussed below. We do not need to make any assumptions about the electronic subsystem's Hamiltonian or the electronic subsystem's state. This Hamiltonian may contain electronelectron interactions and the subsystem may be in an arbitrary stationary state, equilibrium or non-equilibrium. We want to derive kinetic equations for the expectation value of the spin vector $\hat{\vec{I}}\left(\hat{\vec{I}}^{2}=I(I+1)\right)$. To this end, we 
need to take all components of the density matrix into account, not just only the spin vector. Then, as will be seen below, it is advantageous to use the spherical tensor operators $\hat{T}_{L M}$ as a complete basis in the space of operators acting on the state of a spin $I$. The $\hat{T}_{L M}$ are the irreducible tensor operators in the spherical coordinate representation [13]. For actual calculations, the following definition using the spherical harmonics $Y_{L M}$ proves very helpful:

$$
\hat{T}_{L M}:=\mathcal{N}_{I L}(\hat{\vec{I}} \cdot \vec{\nabla})^{L} \quad r^{L} Y_{L M}(\hat{r}) .
$$

$\hat{T}_{L M}$ is a polynomial in the components of the spin operator. It is independent of the auxiliary variable $\vec{r}$, since on the r.h.s., there are $L$ derivatives acting on a polynomial of order $L(\hat{r}=\vec{r} / r$ denotes the unit vector; we use the conventions of Ref. [13] for the spherical harmonics $\left.Y_{L M}\right)$. For a spin $I$, the $(2 I+1)^{2}$ operators $\hat{T}_{L M}$ with $L=0 \cdots 2 I$ and $M=-L \cdots L$ form a complete system of operators acting in the spinspace. The normalization, $\mathcal{N}_{I L}=2^{L} \sqrt{4 \pi(2 I-L) ! /(2 I+L+1) !} / L$ ! , is choosen such that $S p\left\{\hat{T}_{L M}^{\dagger} \hat{T}_{L^{\prime} M^{\prime}}\right\}=\delta_{L L^{\prime}} \delta_{M M^{\prime}}$, and we have $\hat{T}_{L M}^{\dagger}=(-1)^{M} \hat{T}_{L,-M}$. Specific expressions for the operators $\hat{T}_{L, M}$ (for $L=0 \cdots 4$ ) can be found in Table IV of Ref. [14].

After having established the basic notation, we proceed now to describe the derivation of the kinetic equation for the average $T_{L M}(t)=\left\langle\hat{T}_{L M}\right\rangle$, to find the stationary solution and to study finally the relaxation towards the stationary solution. Here and below, the brackets $\langle\cdots\rangle$ stand for the state of the combined system of spin and electrons. We use the framework of the Keldysh method [15] in order to derive the kinetic equation. As in our earlier work on the electron spin relaxation [16], we shall employ the adiabatic approximation; that means that in the equation of motion, the effect of the coupling between spin and electrons is neglected beyond the first order in the spin's eigenenergies and also neglected beyond the second order in the relaxation times, cf. Ref. [17]. The unperturbed motion of the spin is a precession with the frequency $\omega_{0}=\gamma B_{0}$. Then, we have up to the second order of the perturbation theory in the coupling $\hat{V}=-\gamma \hat{\vec{I}} \cdot \hat{\vec{B}}$ :

$$
\begin{aligned}
& \left(i \partial_{t}-M \omega_{0}\right) T_{L M}(t)= \\
& \left\langle\left[\hat{T}_{L M}(t), \hat{V}(t)\right]\right\rangle \\
& -i \int_{-\infty}^{t} d t_{1}\left\langle\left[\left[\hat{T}_{L M}(t), \hat{V}(t)\right], \hat{V}\left(t_{1}\right)\right]\right\rangle+\mathcal{O}\left(\hat{V}^{3}\right) .
\end{aligned}
$$

In the spirit of the adiabatic approximation, we now consider the terms of the perturbation series in higher than second order as giving rise to an additional (weak) time dependence of the spin operators due to the relaxation process, and we decouple the expectation values of spin and electron operators. The first order term,
$\left\langle\left[\hat{T}_{L M}, \hat{\vec{I}}\right](t)\right\rangle \cdot\langle\hat{\vec{B}}(t)\rangle$, describes the Knight shift, the shift of the nuclear resonance frequency due to the coupling to the electrons. Since we want to focus on the relaxation, we disregard this corrections of the spin's eigenfrequency in the following. Then, we get

$$
\begin{aligned}
& \left(i \partial_{t}-M \omega_{0}\right) T_{L M}(t)= \\
& -i \sum_{m ; m^{\prime}=0, \pm 1}\left(\left\langle\left[\left[\hat{T}_{L M}, \hat{I}_{m}\right], \hat{I}_{m^{\prime}}\right](t)\right\rangle C_{-m ;-m^{\prime}}\right. \\
& \left.\quad+\left\langle\left\{\left[\hat{T}_{L M}, \hat{I}_{m}\right], \hat{I}_{m^{\prime}}\right\}(t)\right\rangle R_{-m ;-m^{\prime}}\right)
\end{aligned}
$$

In deriving Eq. (4), we took the unperturbed time dependence of the operator $\hat{\vec{I}}\left(t_{1}\right)$ into account, but neglected the difference $t-t_{1}$ in its time dependence due to the relaxation. In Eq. (州), equal-time commutators ([,]) and anticommutators $(\{\}$,$) can be evaluated, and the expec-$ tation values can again be expressed by $T_{L M}(t)$ as we shall see below. Thus, it is the use of the spherical tensor operators that makes it possible to derive closed coupled equations for the relaxation of the spin. Most crucial are the averages of the electronic subsystem that enter Eq. (4). Since we do not assume thermodynamic equilibrium for the electronic subsystem, we get both, a correlation function and also a response function which are independent and which we denote by $C_{m ; m^{\prime}}$ and $R_{m ; m^{\prime}}$, respectively. They are given by

$$
\begin{aligned}
& C_{m ; m^{\prime}}=\frac{\gamma^{2}}{2} \int_{-\infty}^{t} d t_{1} e^{-i m^{\prime} \omega_{0}\left(t-t_{1}\right)}\left\langle\left\{\hat{B}_{m}(t), \hat{B}_{m^{\prime}}\left(t_{1}\right)\right\}\right\rangle \\
& R_{m ; m^{\prime}}=\frac{\gamma^{2}}{2} \int_{-\infty}^{t} d t_{1} e^{-i m^{\prime} \omega_{0}\left(t-t_{1}\right)}\left\langle\left[\hat{B}_{m}(t), \hat{B}_{m^{\prime}}\left(t_{1}\right)\right]\right\rangle
\end{aligned}
$$

Our convention for the vector components of $\hat{\vec{I}}$ and $\hat{\vec{B}}$ is: $\hat{I}_{ \pm 1}=\hat{I}_{x} \pm i \hat{I}_{y}, \hat{I}_{0}=\hat{I}_{z}$ and $\hat{B}_{ \pm 1}=\left(\hat{B}_{x} \pm i \hat{B}_{y}\right) / 2$, $\hat{B}_{0}=\hat{B}_{z}$.

Commutators and anticommutators in Eq. (4) can be calculated either directly from the definition Eq. (2), or with the aid of the theory of spherical tensors (cf. Ref. [18]). The commutators express the behavior of the $\hat{T}_{L M}$ under rotations:

$$
\begin{aligned}
{\left[\hat{T}_{L M}, \hat{I}_{z}\right] } & =-M \hat{T}_{L M} \\
{\left[\hat{T}_{L M}, \hat{I}_{ \pm}\right] } & =-\sqrt{L(L+1)-M(M \pm 1)} \hat{T}_{L, M \pm 1}
\end{aligned}
$$

The anticommutators can also be expanded in $\hat{T}_{L M}$; specializing the general result containing Racah coefficients (see Eq. (17) of ref. [18]) to our case, we get for $m=0, \pm 1$ :

$$
\left\{\hat{T}_{L M}, \hat{I}_{m}\right\}=a_{L+1 ; M}^{(m)} \hat{T}_{L+1, M+m}+b_{L ; M}^{(m)} \hat{T}_{L-1, M+m}
$$


with the coefficients

$$
\begin{aligned}
& a_{L ; M}^{(0)}=b_{L ; M}^{(0)}=\sqrt{L^{2}-M^{2}} c_{L}, \\
& a_{L ; M}^{( \pm 1)}=b_{L ; M \pm 1}^{(\mp 1)}=\mp \sqrt{(L+1 \pm M)(L \pm M)} c_{L},
\end{aligned}
$$

where

$$
c_{L}=\sqrt{\frac{(2 I+1)^{2}-L^{2}}{(2 L)^{2}-1}} .
$$

Inserting relations (7) and (8) into Eq. (4) solves our task of deriving a closed set of relaxation equations for arbitrary nuclear spin $I$. The equations are linear in the expectation values of the spherical tensor operators $\hat{T}_{L M}$. The properties of the electronic system enter the equations parametrically in the form of correlation functions and response functions. Since the spin vector is given by the $\hat{T}_{1 M}$, a relaxation equation for $\langle\hat{\vec{I}}\rangle$ can be extracted from the system of equations (4). In the case of $I=1 / 2$, this is particularly simple, since $\hat{T}_{L M}=0$ for $L>1$ (and $\hat{T}_{00}=1 / \sqrt{2}$ is constant) and we recover the Bloch equations, see below.

In this general form, the equations (4) are still not very transparent. Therefore, we now make additional assumptions regarding the correlation and response functions entering Eq. (4). The term $\hat{I}_{z} \hat{B}_{z}$ in the perturbation $\hat{V}$ (corresponding to $m, m^{\prime}=0$ in Eq. (4)) just changes the spin's resonance frequency $\omega_{0}$. Since we already omitted the Knight shift, the first order correction term to this frequency, we now also omit consistently the second order contributions resulting from $m=0$ or $m^{\prime}=0$ in Eq. (4). Next, the terms with $m=m^{\prime}$ in Eq. (4) are neglected too. In the electron system, they would correspond to a total change of the $z$-component of the spin by 2 ; if the electrons' state is a strict eigenstate to $z$-component of the spin, such expectation values vanish. Under these assumptions, both correlation and response functions, $C_{m ; m^{\prime}}$ and $R_{m ; m^{\prime}}$, now become "diagonal" in the index $m, C_{m ; m^{\prime}}=\delta_{m ;-m^{\prime}} C_{m}$ and $R_{m ; m^{\prime}}=\delta_{m ;-m^{\prime}} R_{m}$. Now, from the general structure of Eq. (4), it is obvious that the equations do not couple $T_{L M}(t)$ for different $M$. So finally, we arrive at our general result, valid for arbitrary spin $I$ and for a non-equilibrium state of the bath

$$
\begin{aligned}
& \left(\partial_{t}+i M \omega_{0}\right) T_{L M}(t)= \\
& -\frac{1}{2 \tau_{+}}\left[L(L+1)-M^{2}\right] T_{L M}(t) \\
& -\frac{1}{2 \tau_{-}}\left\{L \sqrt{(L+1)^{2}-M^{2}} c_{L+1} T_{L+1, M}(t)\right. \\
& \left.\quad-(L+1) \sqrt{L^{2}-M^{2}} c_{L} T_{L-1, M}(t)\right\} .
\end{aligned}
$$

We have defined two different times by $1 / \tau_{+}:=2\left(C_{1}+\right.$ $\left.C_{-1}\right)$ and $1 / \tau_{-}:=2\left(R_{1}-R_{-1}\right)$. These times are independent, as long as we assume a non-equilibrium state of the electronic system. We have $\tau_{+} \geq 0$ and the ratio $\tau_{+} / \tau_{-}$ can be expressed as $\tau_{+} / \tau_{-}=(1-q) /(1+q)$ with positive $q$. Thus, $\left|\tau_{+} / \tau_{-}\right| \leq 1$. If the electrons are in equilibrium at temperature $T$, the fluctuation-dissipation theorem results in $q=\exp \left(-\omega_{0} / T\right)$.

We want to stress that these relaxation equations are valid (under the assumptions stated above), no matter which specific relaxation mechanism one wants to consider. The special mechanism enters the equations in the form of two time scales, $\tau_{+}$and $\tau_{-}$. In the case of thermodynamic equilibrium in the bath, their ratio, $\tau_{+} / \tau_{-}$, is fixed by the temperature, and only the time $\tau_{+}-$which also depends on temperature - is specific for the relaxation mechanism.

Relaxation equations generally serve two purposes. First, they determine a stationary state. Second, they describe the relaxation towards this state. We now want to discuss both of these points.

Stationary solution

The study of the stationary solution of Eq. (12) serves as an important test of our procedure, since, in the case of equilibrium in the bath, the result is obvious. With the ansatz $T_{L M}(t)=\delta_{M ; 0} T_{L}$, one derives a recursion relation

$c_{L+1} T_{L+1}=-\frac{\tau_{-}}{\tau_{+}} T_{L}+c_{L} T_{L-1}, L=1 \cdots 2 I$

where $T_{0}=(2 I+1)^{-1 / 2}, T_{2 I+1}=0$. The solution is

$$
I_{z}=\frac{2}{3} I(I+1)(1-q) \mathcal{M}_{21} / \mathcal{M}_{11}
$$

where $\mathcal{M}_{i j}$ are elements of the matrix $\mathcal{M}=M_{1}$. $M_{2} \cdots M_{2 I}$ with

$$
M_{L}=\left(\begin{array}{cc}
1+q & (1-q)^{2} \\
1 & 0
\end{array} c_{L+1}^{2}\right)
$$

Evaluation of this matrix product yields - for a general non-equilibrium state of the bath - the following stationary distribution for the $z$-component of the spin vector

$$
I_{z}=\sum_{m=-I}^{I} m q^{-m} / \sum_{m=-I}^{I} q^{-m} .
$$

If we assume now the electronic system is in equilibrium at temperature $T$, we find that $I_{z}$ is given by the Brillouin function, the well-known correct equilibrium distribution for a spin $I$.

\section{Relaxation}

The general solution of the coupled relaxation equations Eq. (12) is a superposition of exponentially decaying terms. We are mostly interested in the relaxation of the spin components $\hat{I}_{z} \propto \hat{T}_{10}$ and $\left(\hat{I}_{x}+i \hat{I}_{y}\right) \propto \hat{T}_{11}$, which are described by the part $M=0$ and $M=1$ of the full system of equations. For $M=0$ and $M=1$, there are $2 I$ different relaxation times, for $M=2$ there are $2 I-1$ 
times; in total we have $I(2 I+3)$ times. Here, each case of $I$ needs to be discussed separately.

We start with spin $1 / 2$. In this case, the Eq. (12) gives immediately the standard Bloch equations [1]:

$$
\begin{gathered}
\partial_{t} I_{z}(t)=-\frac{1}{\tau_{+}}\left(I_{z}(t)-\frac{\tau_{+}}{2 \tau_{-}}\right) \\
\left(\partial_{t}+i \omega_{0}\right) I_{+}(t)=-\frac{1}{2 \tau_{+}} I_{+}(t)
\end{gathered}
$$

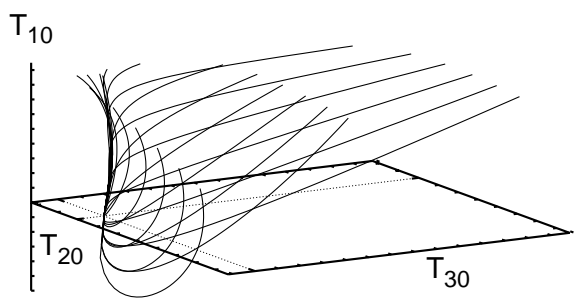

FIG. 1. Spin $I=3 / 2$ : Solution of the relaxation equations for $M=0$ with $\tau_{+} / \tau_{-}=0.8 ; I_{z} \propto T_{10}$ (arbitrary units used).

More interesting, because of its experimental relevance [2], is the case of spin $3 / 2$. The relaxation equations for $M=0$ couple the expectation values of three operators: $\hat{T}_{10}, \hat{T}_{20}$, and $\hat{T}_{30}$. These equations are easily solved. In Fig. 1, we show the flow of the expectation values towards the stationary state at the origin in the case of $\tau_{+} / \tau_{-}=0.8$. The inverse time scales, $\lambda$, which determine the relaxation are shown for both the longitudinal $\left(\lambda_{L}, M=0\right)$ and the transverse equations $\left(\lambda_{T}, M=1\right)$ in units of $1 / \tau_{+}$as functions of the temperature in the case of equilibrium in the bath in Fig. 2. Between the hightemperature limit $T>>\omega_{0}$ and the low-temperature limit $T<<\omega_{0}$, both the largest longitudinal and the largest transverse relaxation time decrease by a factor of three as compared to the relaxation time in Bloch approximation calculated in the usual perturbative way.

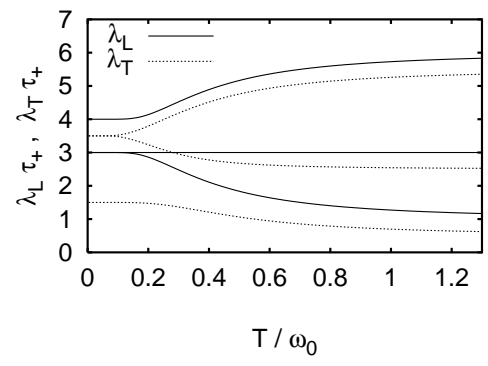

FIG. 2. Spin $I=3 / 2$ : Inverse relaxation times in the longitudinal (solid line) and transverse (dashed line) relaxation equations in units of $1 / \tau_{+}$.

For general $I$, we determine both the longitudinal and the transverse relaxation times in the following limiting cases. For $T>\omega_{0}\left(\tau_{+} / \tau_{-} \sim 0\right)$, the longitudinal relaxation time is $\tau_{+}$and the transverse $2 \tau_{+}$as in the Bloch equations. In the opposite case, $T<<\omega_{0}$ $\left(\tau_{+} / \tau_{-} \rightarrow 1\right)$, all the operators $\hat{T}_{L M}$ become equally important in Eq. (12). Then, the $2 I$ relaxation times are as follows $(n=0,1, \cdots 2 I-1)$ : In the longitudinal case, $M=0$, we get $\tau_{+} /[2(n+1) I-n(n+1)]$ and the longest relaxation time $(n=0)$ is always twofold degenerate; in the transverse case, $M=1$, we get $\tau_{+} /\left[(2 n+1) I-n^{2}\right]$ and here, the longest relaxation time $(n=0)$ is always non-degenerate. These explicite expressions are a conjecture based on an explicite evaluation of the relaxation equations for spin $I$ up to $7 / 2$.

Starting from the microscopic description, we have derived relaxation equations valid for an arbitrary spin $I$ coupled to a quantum mechanical environment in a nonequilibrium state. The solution of these coupled equations shows that, compared to the Bloch equations, there is an additional temperature dependence in the relaxation time which can decrease the relaxation time by a factor of up to $2 I$.

We would like to thank the Deutsche Forschungsgemeinschaft for its support (Ap 47/3-1, 436 RUS 17/73/00). $\mathrm{Yu}$. A.B. wishes to acknowledge support from grants RFFI-00-02-17292, IR-97-0076, and INTAS 99-01146, and wishes to thank the PTB, where this work was performed, for its hospitality.

[1] C. P. Slichter, Principles of Magnetic Resonance, Springer Series in Solid-State Sciences, Vol. 1 (SpringerVerlag, Berlin Heidelberg New York, 1980).

[2] M. Dobers et al., Phys. Rev. Lett. 61, 1650 (1988).

[3] A. Berg, M. Dobers, R. R. Gerhardts, and K. von Klitzing, Phys. Rev. Lett. 64, 2563 (1990).

[4] S. E. Barrett et al., Phys. Rev. Lett. 74, 5112 (1995).

[5] R. Tycko et al., Science 268, 1460 (1995).

[6] P. Khandelwal et al., Phys. Rev. Lett. 81, 673 (1998).

[7] N. N. Kuzma et al., Science 281, 686 (1998).

[8] S. Melinte et al., Phys. Rev. Lett. 84, 354 (2000).

[9] S. M. Girvin, in Topological aspects of low dimensional systems, Les Houches - Ecole d'Ete de Physique Theorique, Session LXIX, edited by A. Comtet, T. J. amd S. Ouvry, and F. David (Springer-Verlag, Berlin/Heidelberg Germany, 1999), Chap. 2, pp. 53-176; also in cond-mat/9907002.

[10] R. Côté et al., Phys. Rev. Lett. 78, 4825 (1997).

[11] J. Sinova, S. M. Girvin, T. Jungwirth, and K. Moon, Phys. Rev. B 61, 2749 (2000).

[12] R. K. Wangsness and F. Bloch, Phys. Rev. 89, 728 (1953).

[13] M. E. Rose, Elementary Theory of Angular Momentum (John Wiley \& Sons, New York, 1957).

[14] E. Ambler, J. C. Eisenstein, and J. F. Schooley, J. Math. Phys. 3, 118 (1962).

[15] L. V. Keldysh, Sov. Phys. JETP 20, 1018 (1965).

[16] W. Apel and Y. A. Bychkov, Phys. Rev. Lett. 82, 3324 (1999).

[17] L. D. Landau and E. M. Lifshitz, in Physical Kinetics, Vol. X of Course of Theoretical Physics (Pergamon, Oxford, 1981), Chap. $\S 91$.

[18] M. E. Rose, J. Math. Phys. 3, 409 (1962). 